TRANSACTIONS OF THE

AMERICAN MATHEMATICAL SOCIETY

Volume 350, Number 5, May 1998, Pages 2033-2048

S 0002-9947(98)02190-4

\title{
EQUIVALENCE OF NORMS ON OPERATOR SPACE TENSOR PRODUCTS OF $C^{*}$-ALGEBRAS
}

\author{
AJAY KUMAR AND ALLAN M. SINCLAIR
}

\begin{abstract}
The Haagerup norm $\|\cdot\|_{h}$ on the tensor product $A \otimes B$ of two $C^{*}$-algebras $A$ and $B$ is shown to be Banach space equivalent to either the Banach space projective norm $\|\cdot\|_{\gamma}$ or the operator space projective norm $\|\cdot\|_{\wedge}$ if and only if either $A$ or $B$ is finite dimensional or $A$ and $B$ are infinite dimensional and subhomogeneous. The Banach space projective norm and the operator space projective norm are equivalent on $A \otimes B$ if and only if $A$ or $B$ is subhomogeneous.
\end{abstract}

\section{INTRODUCTION}

Recently D.P. Blecher and V.I. Paulsen [4], [2], [3] and E.G. Effros and Z-J. Ruan [10], [9], [8] have independently developed the theory of operator spaces with analogous constructions to those of Banach spaces; for example, quotients, duals and tensor products are defined and studied. The Haagerup $\|\cdot\|_{h}$, operator space projective $\|\cdot\|_{\wedge}$ and Banach space projective $\|\cdot\|_{\gamma}$ (tensor) norms on the tensor product $A \otimes B$ of two $C^{*}$-algebras $A$ and $B$ satisfy

$$
\|\cdot\|_{h} \leq\|\cdot\|_{\wedge} \leq\|\cdot\|_{\gamma} .
$$

For commutative $C^{*}$-algebras $A$ and $B$ one version of the commutative Grothendieck inequality [22], [6] is that $\|\cdot\|_{\gamma} \leq K\|\cdot\|_{h}$, where $K$ is Grothendieck's constant. This raises the question: for which pairs of $C^{*}$-algebras $A$ and $B$ are these tensor norms equivalent? Itoh ([14], Theorem 4 ) claims that the Haagerup norm $\|\cdot\|_{h}$ and Banach space projective norm $\|\cdot\|_{\gamma}$ are equivalent on the algebraic tensor product $A \otimes B$ of two $C^{*}$-algebras $A$ and $B$ if and only if $A$ or $B$ is subhomogeneous. This result is false as can be seen by considering these two norms on $\ell^{\infty} \otimes B(H)$, or $\ell^{\infty} \otimes K(H)$, where $H$ is an infinite dimensional Hilbert space, and $B(H)$ and $K(H)$ denote the bounded linear operators on $H$ and the compact linear operators on $H$.

The correct version (Theorem 6.1) of this result is: the Haagerup norm and Banach space projective norm are equivalent on the algebraic tensor product $A \otimes B$ of two $C^{*}$-algebras $A$ and $B$ if, and only if, either $A$ or $B$ is finite dimensional, or $A$ and $B$ are infinite dimensional and subhomogeneous. The same conclusion holds if the Banach space projective norm is replaced by the operator space projective norm above (Theorem 7.4). The latter result is based on the equivalence of the

Received by the editors August 16, 1996.

1991 Mathematics Subject Classification. Primary 46L05; Secondary 46C10, 47035.

Key words and phrases. Banach space projective norm, operator space projective norm, Haagerup norm, $C^{*}$-algebras, second dual.

Supported by Commonwealth Academic Staff Fellowship at the University of Edinburgh. 
Banach space projective norm and operator space projective norm (Theorem 7.2), which hold if and only if $A$ or $B$ is subhomogeneous. Thus both hypotheses in Itoh have a role here.

Our methods involve the commutative and non-commutative Grothendieck inequalities, lifting maps to second duals and to tensor products of second duals (see sections 4 and 5) and elementary calculations involving $\ell_{n}^{\infty}$ and $\mathbb{M}_{n}$. Section 2 contains the notation and definitions used in the paper, and section 3 has the matrix calculations and a helpful observation concerning an isometric involution on the operator space projective tensor product $A \widehat{\otimes} B$ of two $C^{*}$-algebras $A$ and $B$. The well known self-duality and injectivity of the Haagerup tensor product [4], [9], [3] is presented in a slightly modified form in Theorem 4.1. This forms a crucial basis of sections 6 and 7, and is a motivation for proving the corresponding result (Theorem 5.1) for the Banach space projective tensor. We have not been able to prove an equivalent result for the operator space projective tensor norm due to not knowing if the natural embedding of $A^{* *} \widehat{\otimes} B^{* *}$ into $(A \widehat{\otimes} B)^{* *}$ is isometric (or has continuous inverse). R.J. Archbold and C.J.K. Batty [1] defined property $(C)$ for a $C^{*}$-algebra $A$ to be the condition that $A^{* *} \otimes_{\min } B^{* *}$ canonically embed in $\left(A \otimes_{\min } B\right)^{* *}$. This is clearly related to our results for $\otimes_{h}$ and $\otimes_{\gamma}$, and what is needed for $\widehat{\otimes}$. The equivalence of the Haagerup norm and the Banach space projective norm is handled in section 6 with the two equivalences involving the operator space projective norm covered in section 7 .

We wish to thank Christian le Merdy for discussions concerning the operator space projective norm and Roger Smith for drawing [1] to our attention.

\section{Notation AND DEFinitions}

An operator space $X$ is a (norm closed) subspace of $B(H)$ together with the natural norms on $\mathbb{M}_{n}(X)$ inherited from $\mathbb{M}_{n}(B(H))=B\left(H^{n}\right)$, where $H$ is a Hilbert space and $H^{n}$ is the $n$-fold Hilbert space direct sum of $H$ with itself. An operator $\phi: X \rightarrow Y$, between operator spaces $X$ and $Y$ is completely bounded if and only if $\|\phi\|_{c b}=\sup \left\{\left\|\phi_{n}\right\|: n \in \mathbb{N}\right\}$ is finite, where $\phi_{n}: \mathbb{M}_{n}(X) \rightarrow \mathbb{M}_{n}(Y)$ is defined by $\phi_{n}\left(x_{i j}\right)=\left(\phi x_{i j}\right)$. Let $C B(X, Y)$ denote the space of completely bounded operators from $X$ into $Y$ [19].

Throughout let $A$ and $B$ denote $C^{*}$-algebras. The Haagerup tensor norm $\|u\|_{h}$ of an element $u$ in the algebraic tensor product $A \otimes B$ is defined by

$$
\|u\|_{h}=\inf \left\{\left\|\sum a_{j} a_{j}^{*}\right\|^{\frac{1}{2}}\left\|\sum b_{j}^{*} b_{j}\right\|^{\frac{1}{2}}: u=\sum a_{j} \otimes b_{j} \in A \otimes B\right\},
$$

and the Haagerup tensor product $A \otimes_{h} B$ is the completion of $A \otimes B$ in this norm. If $p$ and $q$ are positive integers, and $V=\left(v_{i j}\right) \in \mathbb{M}_{p}(A)$ and $W=\left(w_{i j}\right) \in \mathbb{M}_{q}(B)$ define the tensor product $V \otimes W$ to be the $p q \times p q$ matrix

$$
V \otimes W=\left(v_{i j} \otimes w_{k \ell}\right) \text { in } \mathbb{M}_{p q}(A \otimes B)
$$

using $\left(\mathbb{M}_{p} \otimes A\right) \otimes\left(\mathbb{M}_{q} \otimes B\right) \cong \mathbb{M}_{p q} \otimes A \otimes B$. Each element $u$ in $A \otimes B$ can be written $u=\alpha(V \otimes W) \beta$ for some $\alpha \in \mathbb{M}_{1, p q}, V \in \mathbb{M}_{p}(A), W \in \mathbb{M}_{q}(B)$ and $\beta \in \mathbb{M}_{p q, 1}$ where $\mathbb{M}_{k, \ell}$ is the space of complex $k \times \ell$ matrices over $\mathbb{C}$. The operator space projective (tensor) norm $\|u\|_{\wedge}$ is defined on the algebraic tensor $A \otimes B$ by

$\|u\|_{\wedge}=\inf \{\|\alpha\| \cdot\|V\| \cdot\|W\| \cdot\|\beta\|: u=\alpha(V \otimes W) \beta$ is a decomposition as above $\}$, 
and the operator space projective tensor product $A \widehat{\otimes} B$ is the completion of $A \otimes B$ in this norm [4], [10]. Recall that the Banach space projective (tensor) norm $\|\cdot\|_{\gamma}$ is defined on the algebraic tensor product $X \otimes Y$ of two Banach spaces $X$ and $Y$ by

$$
\|u\|_{\gamma}=\inf \left\{\sum\left\|x_{j}\right\| \cdot\left\|y_{j}\right\|: u=\sum x_{j} \otimes y_{j} \in X \otimes Y\right\},
$$

and that $X \otimes_{\gamma} Y$ is the completion of $X \otimes Y$ under this tensor product. It is well known that $\|\cdot\|_{h} \leq\|\cdot\|_{\wedge} \leq\|\cdot\|_{\gamma}$ on the tensor product of two $C^{*}$-algebras.

Recall that a $C^{*}$-algebra $A$ is subhomogeneous if there is a positive integer $n$ such that each irreducible representation of $A$ has dimension no greater than $n$. Further that $A$ is subhomogeneous with all its irreducible representations of dimension no greater than $n$ if and only if its enveloping von Neumann algebra $A^{* *}$ is finite type I with all its central direct summands of type no greater than $\mathrm{I}_{n}$.

A basic technique used in this paper is to pass from inequalities involving tensor norms on von Neumann algebras to the tensor norms of finite dimensional subalgebras. The injectivity of the Haagerup tensor product [20] implies that the two norms are equal in that case. If $A_{0}$ and $B_{0}$ are finite dimensional $C^{*}$-algebras of $A$ and $B$, respectively, then there are completely positive conditional expectations $P_{1}$ and $P_{2}$ from $A$ and $B$ onto $A_{0}$ and $B_{0}$, respectively, and $\left\|P_{1}\right\|_{c b}=\left\|P_{2}\right\|_{c b}=1$ [26]. Hence $P_{1} \otimes P_{2}$ is a projection from $A \otimes B$ onto $A_{0} \otimes B_{0}$ such that $\left\|P_{1} \otimes P_{2}\right\|=1$ when both tensor products are given either the Banach space $\|\cdot\|_{\gamma}$ or operator space $\|\cdot\|_{\wedge}$ projective norms. This implies that the natural isomorphism from $A_{0} \otimes B_{0}$ into $A \otimes B$ is an isometry in both these norms.

\section{BASIC LEMMAS}

This section contains the lemmas dealing with the crucial matrix calculations involving $\|\cdot\|_{h},\|\cdot\|_{\wedge}$ and $\|\cdot\|_{\gamma}$. Denote the standard matrix units in $\mathbb{M}_{n}$ by $e_{i j}$ for $1 \leq i, j \leq n$ and identify the $n$-dimensional $L^{\infty}$-space $\ell_{n}^{\infty}$ with the diagonal of $\mathbb{M}_{n}$ so that it has standard basis $\left\{e_{j j}: 1 \leq j \leq n\right\}$.

Lemma 3.1. If $n \in \mathbb{N}$, then

(i)

$$
\left\|\sum_{1}^{n} e_{1 j} \otimes e_{j j}\right\|_{h}=n^{\frac{1}{2}} \text { and }\left\|\sum_{1}^{n} e_{j 1} \otimes e_{j j}\right\|_{h}=1
$$

(ii)

in $\mathbb{M}_{n} \otimes_{h} \ell_{n}^{\infty}$,

$$
\left\|\sum_{1}^{n} e_{j j} \otimes e_{j 1}\right\|_{h}=n^{\frac{1}{2}} \text { and }\left\|\sum_{1}^{n} e_{j j} \otimes e_{1 j}\right\|_{h}=1
$$

in $\ell_{n}^{\infty} \otimes_{h} \mathbb{M}_{n}$ and

$$
\left\|\sum e_{1 j} \otimes e_{j 1}\right\|_{h}=n
$$

in $\mathbb{M}_{n} \otimes_{h} \mathbb{M}_{n}$.

Proof. (i) It is sufficient to prove the first pair of equalities in $\mathbb{M}_{n} \otimes_{h} \ell_{n}^{\infty}$ because the second pair follow directly using the conjugate linear isometry $\theta$ from $\mathbb{M}_{n} \otimes_{h} \ell_{n}^{\infty}$ onto $\ell_{n}^{\infty} \otimes_{h} \mathbb{M}_{n}$ defined by $\theta(x \otimes y)=y^{*} \otimes x^{*}[17]$. 
Let $u=\sum_{1}^{n} e_{1 j} \otimes e_{j j}$. By definition of the Haagerup norm,

$$
\|u\|_{h}^{2} \leq\left\|\sum_{1}^{n} e_{1 j} e_{j 1}\right\| \cdot\left\|\sum_{1}^{n} e_{j j} e_{j j}\right\|=n .
$$

A standard Cauchy-Schwarz argument that occurs in Haagerup's unpublished paper on this tensor product implies that the map

$$
\pi: \mathbb{M}_{n} \otimes_{h} \mathbb{M}_{n} \rightarrow B\left(\mathbb{M}_{n}\right)
$$

defined by $\pi(x \otimes y)(a)=x a y$ is norm reducing; it is actually a complete isometry into $C B\left(\mathbb{M}_{n}\right)$ (see [25], [18]).

Hence $\|u\|_{h} \geq\|\pi(u)(I)\|=\left\|\sum e_{1 j} e_{j j}\right\|=\left\|\sum_{i, j} e_{1 j} e_{i 1}\right\|^{\frac{1}{2}}=n^{\frac{1}{2}}$ so proving the first equality.

(ii) The second equality follows from

$$
\begin{aligned}
1 & =\left\|\sum e_{j 1} e_{j j}\right\| \\
& =\left\|\pi\left(\sum e_{j 1} \otimes e_{j j}\right)(I)\right\| \\
& \leq\left\|\sum e_{j 1} \otimes e_{j j}\right\|_{h} \\
& \leq\left\|\sum e_{j 1} e_{j 1}^{*}\right\|^{\frac{1}{2}} \cdot\left\|\sum_{1}^{n} e_{j j} e_{j j}\right\|^{\frac{1}{2}}=1 .
\end{aligned}
$$

(iii) The third inequality follows from

$$
\left\|\sum_{1}^{n} e_{1 j} \otimes e_{j 1}\right\|_{h}^{2} \leq\left\|\sum_{1}^{n} e_{1 j} e_{j 1}\right\| \cdot\left\|\sum_{1}^{n} e_{1 j} e_{j 1}\right\|=n^{2}
$$

and

$$
\left\|\sum_{1}^{n} e_{1 j} \otimes e_{j 1}\right\|_{h} \geq\left\|\pi\left(\sum_{1}^{n} e_{1 j} \otimes e_{j 1}\right)(I)\right\|=n .
$$

Lemma 3.2. Let $A, B, C, D$ be $C^{*}$-algebras.

(i) If $\theta$ is a $*$-anti-homomorphism from $A$ into $C$ and $\phi$ is $a$-anti-homomorphism from $B$ into $D$, then $\theta \otimes \phi$ is a $*$-anti-homomorphism from $A \widehat{\otimes} B$ into $C \widehat{\otimes} D$ with $\|\theta \otimes \phi\|=1$.

(ii) If the involution $*$ is defined on $A \otimes B$ by $(a \otimes b)^{*}=a^{*} \otimes b^{*}$, then $*$ lifts to an isometric involution on $A \widehat{\otimes} B$.

Proof. (i) Once $\|\theta \otimes \phi\| \leq 1$ has been proved the other properties follow directly; note the norm equals 1 except when $\theta$ or $\phi$ is zero. Let $u=\alpha(V \otimes W) \beta$ be in $A \otimes B$ with $\alpha \in \mathbb{M}_{1, p q}, V \in \mathbb{M}_{p}(A), W \in \mathbb{M}_{q}(B), \beta \in \mathbb{M}_{p q, 1}$ and $\|\alpha\| \cdot\|V\| \cdot\|W\| \cdot\|\beta\| \leq$ 1.

If $T$ denotes the transpose, then

$$
\begin{aligned}
(\theta \otimes \phi)(u) & =\sum \alpha_{i s} \theta\left(v_{i j}\right) \otimes \phi\left(w_{s t}\right) \beta_{j t} \\
& =\sum \beta_{j t}(T \otimes I) \theta_{n}(V)_{j i} \otimes(T \otimes I) \phi_{n}(W)_{t s} \alpha_{i s} \\
& =(T \beta) .(T \otimes I) \theta_{n}(V) \otimes(T \otimes I) \phi_{n}(W)(T \alpha) .
\end{aligned}
$$


Here $(T \otimes I) \theta_{p}$ and $(T \otimes I) \phi_{q}$ are $*$-anti-homomorphisms from $\mathbb{M}_{p}(A)$ into $\mathbb{M}_{p}(C)$ and $\mathbb{M}_{q}(B)$ into $\mathbb{M}_{q}(D)$, respectively, so have norm 1. Thus

$$
\begin{aligned}
& \|T \beta\| \cdot\left\|(T \otimes I) \theta_{p} V\right\| \cdot\left\|(T \otimes I) \phi_{q} W\right\| \cdot\|T \alpha\| \\
& \quad=\|\beta\| \cdot\|V\| \cdot\|W\| \cdot\|\alpha\| \\
& \quad \leq 1
\end{aligned}
$$

and $T \beta,(T \otimes I) \theta_{p} V,(T \otimes I) \phi_{q} W, T \alpha$ are in the correct matrix spaces. This proves that $\|\theta \otimes \phi(u)\| \leq 1$ if $\|u\|_{\wedge}<1$, and so $\|\phi \otimes \phi\|_{\wedge} \leq 1$ as required. The reverse inequality is achieved on elementary tensors.

(ii) If $u$ is in $A \otimes B$ with $u=\alpha(V \otimes W) \beta$ as in (i), then

$$
u^{*}=\beta^{*}\left(V^{*} \otimes W^{*}\right) \alpha^{*}
$$

as may be checked by writing out both expressions in full. Thus $\left\|u^{*}\right\|_{\wedge} \leq 1$ if $\|u\|_{\wedge}<1$ so proving (ii).

Note that the same technique but including the "canonical" shuffle exploited by Paulsen [19] may be used to prove the well known commutativity of $\widehat{\otimes}[4]$, [9].

Lemma 3.3. If $n \in \mathbb{N}$, then in $\mathbb{M}_{n} \otimes \mathbb{M}_{n}$

(i) $\left\|\sum e_{j 1} \otimes e_{j 1}\right\|_{\wedge}=\left\|\sum e_{1 j} \otimes e_{1 j}\right\|_{\wedge}=n^{\frac{1}{2}}$,

(ii) $\left\|\sum e_{1 j} \otimes e_{j 1}\right\|_{\wedge}=\left\|\sum e_{j 1} \otimes e_{1 j}\right\|_{\wedge}=n$, and

(iii) $\left\|\sum e_{j 1} \otimes e_{j 1}\right\|_{\gamma}=n$.

Proof. By Lemma 3.2 it is sufficient to prove that one of the norms equals $n^{\frac{1}{2}}$ or $n$ in each case.

(i) Let $\alpha$ be the $1 \times n^{2}$ matrix consisting of a $n$-string of one followed by $(n-1)$ zeros so

$$
\alpha=(10 \ldots 010 \ldots 01 \ldots 0)
$$

and let $\beta$ be the $n^{2} \times 1$ matrix with a one in the $(1,1)$ position and all the other entries zero. Let $V$ be the $n \times n$ matrix in $\mathbb{M}_{n}\left(\mathbb{M}_{n}\right)$ with first row

$$
e_{11}, e_{21}, \ldots, e_{n 1}
$$

and all other entries zero. Note that $\|\alpha\|=n^{\frac{1}{2}},\|V\|=1$ and $\|\beta\|=1$, which are calculated using $\|x\|^{2}=\left\|x x^{*}\right\|=\left\|x^{*} x\right\|$. Since

$$
\begin{gathered}
\sum e_{j 1} \otimes e_{j 1}=\alpha V \otimes V \beta, \\
\left\|\sum e_{j 1} \otimes e_{j 1}\right\|_{\wedge} \leq n^{\frac{1}{2}} .
\end{gathered}
$$

The projective operator space norm $\|\cdot\|_{\wedge}$ is greater than the spatial tensor product norm $\|\cdot\|_{*}$ of the two $C^{*}$-algebras [4]. Hence

$$
\begin{aligned}
\left\|\sum e_{j 1} \otimes e_{j 1}\right\|_{\wedge}^{2} & \geq\left\|\sum_{j, k}\left(e_{1 j} \otimes e_{1 j}\right)\left(e_{k 1} \otimes e_{k 1}\right)\right\|_{*} \\
& =n\left\|e_{11} \otimes e_{11}\right\|_{*} \\
& =n
\end{aligned}
$$

using $\left\|x^{*} x\right\|=\|x\|^{2}$. This proves (i).

(ii) The inequality $\|\cdot\|_{\wedge} \geq\|\cdot\|_{h}$ and Lemma 3.1 imply that

$$
\left\|\sum e_{1 j} \otimes e_{j 1}\right\|_{\wedge} \geq\left\|\sum e_{1 j} \otimes e_{j 1}\right\|_{h}=n
$$

and the reverse inequality is trivial. 
(iii) Since the map $T \otimes I$ is an isometry on $\mathbb{M}_{n} \otimes_{\gamma} \mathbb{M}_{n}$,

$$
\begin{aligned}
n & \geq\left\|\sum e_{j 1} \otimes e_{j 1}\right\|_{\gamma} \\
& \geq\left\|\sum e_{1 j} \otimes e_{j 1}\right\|_{\wedge} \\
& =n . \quad \square
\end{aligned}
$$

Observe that the above results are essentially in [9] in dual form: they show that $C B\left(C_{n}, C_{n}\right)=\mathbb{M}_{n}$ and $C B\left(R_{n}, C_{n}\right)$ is the $n \times n$ Hilbert-Schmidt matrices, where $C_{n}$ and $R_{n}$ are $n$ dimensional column and row space respectively.

\section{Embedding HaAgerup tensor PRoducts into SECOND duals}

The theorem in this section summarises and modifies a result of Blecher and Paulsen [4] and Effros and Ruan [9] in a suitable way for subsequent use here. The similarity between the Haagerup tensor product results here and those for the projective tensor product in the next section will also be clearer.

Let $i_{A}$ and $i_{B}$ denote the embeddings of $A$ and $B$ into $A^{* *}$ and $B^{* *}$, respectively. If $\phi$ is in $\left(A \otimes_{h} B\right)^{*}$, then there are cyclic representations $\pi$ and $\theta$ of $A$ and $B$ on Hilbert spaces $H$ and $K$ with unit cyclic vectors $\xi$ and $\eta$, respectively, and a continuous linear operator $T$ from $K$ to $H$ such that

$$
\phi(a, b)=\langle\pi(a) T \theta(b) \eta, \xi\rangle \quad(a \in A, b \in B)
$$

and $\|T\|=\|\phi\|[7]$.

Cyclic representations of $A$ and $B$ lift directly to cyclic representations of the enveloping von Neumann algebras $A^{* *}$ and $B^{* *}$, respectively. This provides a natural lifting of $\phi$ to a continuous linear form $\tilde{\phi}$ on $A^{* *} \otimes_{h} B^{* *}$ with $\|\tilde{\phi}\|=\|\phi\|$ and $\tilde{\phi}$ equal to $\phi$ on $A \otimes_{h} B$. Observe that the operator from $\left(A \otimes_{h} B\right)^{*}$ into $\left(A^{* *} \otimes_{h} B^{* *}\right)^{*}$ defined above is well defined and a linear isometry. The reason is that for fixed $a$ in $A$ and two representations of the same $\phi$ the functionals

$$
b^{* *} \mapsto\left\langle\pi_{j}(a) T_{j} \theta_{j}\left(b^{* *}\right) \eta_{j}, \xi_{j}\right\rangle \quad(j=1,2)
$$

are equal on $B$ and ultraweakly continuous on $B^{* *}$ so are equal on $B^{* *}$ by the Kaplansky Density Theorem. Now holding $b^{* *}$ in $B^{* *}$ fixed and varying $a^{* *}$ in $A^{* *}$ shows, in the same way, that the map $\phi \mapsto \tilde{\phi}$ is well defined. The linearity follows directly from this and direct sum techniques in a standard way. Now define $\zeta$ from $A^{* *} \otimes_{h} B^{* *}$ into $\left(A \otimes_{h} B\right)^{* *}$ by

$$
\zeta\left(a^{* *} \otimes b^{* *}\right)(\phi)=\tilde{\phi}\left(a^{* *} \otimes b^{* *}\right)
$$

for all $\phi$ in $\left(A \otimes_{h} B\right)^{*}$. Then $\zeta$ is a norm reducing operator from $A^{* *} \otimes_{h} B^{* *}$ into $\left(A \otimes_{h} B\right)^{* *}$. It is known that $\zeta$ is an isometry [11, Theorem 3.2]; we show that this may be deduced from the well known isometry from $B(H) \otimes_{h} B(H)$ into $C B(K(H))$.

Theorem 4.1. Let $A$ and $B$ be $C^{*}$-algebras. The embedding $\nu=i_{A} \otimes i_{B}$ of $A \otimes_{h} B$ into $A^{* *} \otimes_{h} B^{* *}$ is an isometry and the embedding $\zeta$ of $A^{* *} \otimes_{h} B^{* *}$ into $\left(A \otimes_{h} B\right)^{* *}$ is an isometry. Further $\zeta \nu$ is the natural embedding $i_{A \otimes_{h} B}$ of $A \otimes_{h} B$ in its second dual $\left(A \otimes_{h} B\right)^{* *}$.

Proof. The injectivity of the Haagerup tensor product ensures that the embedding $\nu$ of $A \otimes_{h} B$ into $A^{* *} \otimes_{h} B^{* *}$ is an isometry [20]. That the map $\zeta$ is an isometry of $A^{* *} \otimes_{h} B^{* *}$ into $\left(A \otimes_{h} B\right)^{* *}$ follows from [11, Theorem 3.2] and the embedding 
of the Haagerup tensor product $A^{* *} \otimes_{h} B^{* *}$ in the tensor $A^{* *} \stackrel{e h}{\otimes} B^{* *}$ of that paper. Alternatively this may be proved directly in this case from the representation of $B(H) \otimes_{h} B(H)$ in $C B(K(H))$, where $K(H)$ denotes the algebra of compact operators on $H$ [25, Theorem 4.3]. Let $A$ and $B$ act as $C^{*}$-algebras in their universal representations on Hilbert spaces $H_{A}$ and $H_{B}$ so that $A^{* *}$ and $B^{* *}$ are isomorphic to the weak-closures of $A$ and $B$ in these representations. By the injectivity of the Haagerup tensor and the two Hilbert space versions of [25, Theorem 4.3], which can easily be deduced from that result, the map $\theta$ from $A^{* *} \otimes_{h} B^{* *}$ into $C B\left(K\left(H_{B}, H_{A}\right)\right)$ defined by $\theta\left(a^{* *} \otimes b^{* *}\right)(T)=a^{* *} T b^{* *}$ is an isometry. Thus if $u=\sum_{\ell} a_{\ell}^{* *} \otimes b_{\ell}^{* *}$ is in $A^{* *} \otimes_{h} B^{* *}$ with $\|u\|_{h}>1$, there is an integer $n$ and a compact operator $S=\left(s_{i j}\right)$ from $\left(H_{A}\right)^{n}$ to $\left(H_{B}\right)^{n}$ with $\|S\|=1$ such that

$$
\left\|\left(\sum_{\ell} a_{\ell}^{* *} s_{i j} b_{\ell}^{* *}\right)\right\|>1 .
$$

There are then unit vectors $\left(\xi_{j}\right)$ in $\left(H_{B}\right)^{n}$ and $\left(\eta_{i}\right)$ in $\left(H_{A}\right)^{n}$ such that

$$
\left|\sum_{\ell} \sum_{i, j}\left\langle a_{\ell}^{* *} s_{i j} b_{\ell}^{* *} \xi_{j}, \eta_{i}\right\rangle\right|>1 .
$$

If

$$
\phi\left(a^{* *} \otimes b^{* *}\right)=\sum_{i, j}\left\langle a^{* *} s_{i j} b^{* *} \xi_{j}, \eta_{i}\right\rangle,
$$

then $\phi$ is in $\left(A^{* *} \otimes_{h} B^{* *}\right)^{*}$ also $\|\phi\| \leq 1$ in $\left(A \otimes_{h} B\right)^{*}$, and $|\phi(u)|>1$. This proves that $\|\zeta(u)\|>1$ and so shows that $\zeta$ is an isometry.

Let $a \otimes b$ be in $A \otimes_{h} B$ and let $\phi$ be in $\left(A \otimes_{h} B\right)^{*}$. Then from the definitions of $\zeta$ and $\nu$

$$
\zeta \nu(a \otimes b)(\phi)=\phi(a \otimes b)=i_{A \otimes_{h} B}(a \otimes b)(\phi)
$$

as required.

\section{Embedding Banach PROJective tensor products of $C^{*}$-Algebras INTO SECOND DUALS}

Let $A$ and $B$ be $C^{*}$-algebras. There is a natural mapping $\nu$ from $A \otimes_{\gamma} B$ into $A^{* *} \otimes_{\gamma} B^{* *}$ that is the tensor product $i_{A} \otimes i_{B}$ of the natural embedding $i_{A}$ of $A$ in $A^{* *}$ and $i_{B}$ of $B$ in $B^{* *}$. The natural embedding of $A^{* *} \otimes_{\gamma} B^{* *}$ into $\left(A \otimes_{\gamma} B\right)^{* *}$ requires a little more explanation. U. Haagerup proved that each continuous bounded bilinear form $\phi$ on a pair of $C^{*}$-algebras $A$ and $B$ can be extended to a jointly ultrastrong-* continuous bilinear form $\tilde{\phi}$ from $A^{* *} \times B^{* *}$ into $\mathbb{C}$ with $\|\tilde{\phi}\|=\|\phi\|[12$, Cor. 2.4]. The extension is achieved by using [15, Lemma 2.1] (see also [23, Lemmas 3.3.2 and 3.3.3]) which ensures that map $\chi \phi=\tilde{\phi}$ is linear and an isometry. The dual of the operator

$$
\chi: \operatorname{Bil}(A \times B, \mathbb{C}) \cong\left(A \otimes_{\gamma} B\right)^{*} \rightarrow \operatorname{Bil}\left(A^{* *} \times B^{* *}, \mathbb{C}\right) \cong\left(A^{* *} \otimes_{\gamma} B^{* *}\right)^{*}
$$

$\operatorname{maps}\left(A^{* *} \otimes_{\gamma} B^{* *}\right)^{* *}$ into $\left(A \otimes_{\gamma} B\right)^{* *}$. Let $\mu=\chi^{*} i$ be the natural norm reducing operator from $A^{* *} \otimes_{\gamma} B^{* *}$ into $\left(A \otimes_{\gamma} B\right)^{* *}$, where $i$ is the natural embedding of $A^{* *} \otimes_{\gamma} B^{* *}$ into $\left(A^{* *} \otimes_{\gamma} B^{* *}\right)^{* *}$. 
Theorem 5.1. Let $A$ and $B$ be $C^{*}$-algebras. The embedding $\nu=i_{A} \otimes i_{B}$ of $A \otimes_{\gamma} B$ into $A^{* *} \otimes_{\gamma} B^{* *}$ is an isometry and the embedding $\mu$ of $A^{* *} \otimes_{\gamma} B^{* *}$ into $\left(A \otimes_{\gamma} B\right)^{* *}$ satisfies

$$
\|u\| \leq 4\|\mu(u)\| \leq 4\|u\|
$$

for all $u$ in $A^{* *} \otimes_{\gamma} B^{* *}$. Further $\mu \nu$ is the natural embedding $i_{A_{\gamma_{\gamma}} B}$ of $A \otimes_{\gamma} B$ in its second dual $\left(A \otimes_{\gamma} B\right)^{* *}$.

The following is a consequence of the non-commutative Grothendieck-PisierHaagerup inequality [12, Lemma Cor 2.4]; we do not know an explicit reference so give a proof.

Lemma 5.2. Let $A$ and $B$ be $C^{*}$-algebras. The natural embedding $\nu=i_{A} \otimes i_{B}$ of $A \otimes_{\gamma} B$ into $A^{* *} \otimes_{\gamma} B^{* *}$ is an isometry.

Proof. The definition of the projective tensor norm ensures that $\|\nu\|=1$. If $u$ is in $A \otimes_{\gamma} B$, there is a continuous bilinear form $\phi$ on $A \otimes B$ with $\|\phi\|=1$ and $\phi(u)=\|u\|$. The extension $\tilde{\phi}$ of $\phi$ to $A^{* *} \times B^{* *}$ referred to above [12, Cor. 2.4] has $\|\tilde{\phi}\|=\|\phi\|$ so that $\|\nu(u)\|=\|u\|$ as required.

The following lemma is equivalent to the theorem that there is an isometric embedding of $A \otimes_{h} B$ into $C B(B(H))$ or $C B(K(H))$ and it will be deduced below from this well known isometry. Actually this lemma occurs in U. Haagerup's unpublished 1981 manuscript "The $a$-tensor product", where it is used to prove the isometry of the embedding of $A \otimes_{h} B$ in $C B(B(H))$.

Lemma 5.3. Let $A$ and $B$ be $C^{*}$-algebras acting on Hilbert spaces $H$ and $K$, respectively. The unit ball of $C B_{\sigma}(A \times B, \mathbb{C})$ is weak*-dense in the unit ball of $C B(A \times B, \mathbb{C})$.

Proof. Suppose the result is not true. By the Hahn-Banach separation theorem there is a $\phi$ in $C B(A \times B, \mathbb{C})$ with $\|\phi\|_{c b}=1$, a $0<\alpha<1$ and a $u$ in $A \otimes_{h} B=$ $C B(A \times B, \mathbb{C})_{*}$ such that $\phi(u)=1$ and $\Re \psi(u) \leq \alpha$ for all $\psi$ in $C B_{\sigma}(A \times B, \mathbb{C})$ with $\|\psi\|_{c b} \leq 1$. Hence $\|u\|_{h} \geq 1$ and $|\psi(u)| \leq \alpha$ for all $\psi$ in $C B_{\sigma}(A \times B, \mathbb{C})$ with $\|\psi\|_{c b} \leq 1$. The $\theta$ embedding from $A \otimes_{h} B$ into $C B(B(H, K))$ given by $\theta(a \otimes b)(T)=$ $a T b$ is an isometry [25], [18]. Hence there is a positive integer $n$ and a matrix $\left(t_{i j}\right) \in \mathbb{M}_{n}(B(H, K))$ with $\left\|\left(t_{i j}\right)\right\|=1$ and

$$
\left\|\theta(u)_{n}\left(t_{i j}\right)\right\|=\left\|\left(\theta(u) t_{i j}\right)\right\|>\alpha .
$$

Choose unit vectors $\xi$ in $K^{n}$ and $\eta$ in $H^{n}$ such that

$$
\left|\left\langle\left(\theta(u) t_{i j}\right) \xi, \eta\right\rangle\right|>\alpha \text {. }
$$

Define $\psi$ in $C B_{\sigma}(A \times B, \mathbb{C})$ by

$$
\psi(a \otimes b)=\psi(a, b)=\left\langle\left(\theta(a \otimes b) t_{i j}\right) \xi, \eta\right\rangle
$$

so that $|\psi(u)|>\alpha$.

Then $\|\psi\|_{c b} \leq\|\xi\| \cdot\left\|\left(t_{i j}\right)\right\|\|\eta\|=1$ because $\psi(a, b)$ can be written

$$
\psi(a, b)=\left\langle(a \otimes I)\left(t_{i j}\right)(b \otimes I) \xi, \eta\right\rangle .
$$

The contradictory inequalities

$$
|\psi(u)|>\alpha \text { and }|\psi(u)| \leq \alpha
$$

imply the result. 
The non-commutative Grothendieck inequality [12], [21], [22] states that if $\phi$ is a continuous bilinear form on $A \times B$, where $A$ and $B$ are $C^{*}$-algebras, then there are states $f_{1}, f_{2}$ and $g_{1}, g_{2}$ on $A$ and $B$, respectively, such that

$$
|\phi(a, b)| \leq\|\phi\|\left(f_{1}\left(x^{*} x\right)+f_{2}\left(x x^{*}\right)\right)^{\frac{1}{2}}\left(g_{1}\left(y^{*} y\right)+g_{2}\left(y y^{*}\right)\right)^{\frac{1}{2}}
$$

for all $x \in A$ and $y \in B$. We now briefly review the construction of the four bilinear forms $\phi_{1}, \ldots, \phi_{4}$ that are continuous in the Haagerup tensor norm, ..., doubly reversed Haagerup tensor norm ([16, Remark 5.3]). Let $\left(\theta_{1}, H_{1}, \eta_{1}\right)$ and $\left(\theta_{2}, H_{2}, \eta_{2}\right)$ be the cyclic representation and cyclic anti-representation derived from $f_{1}$ and $f_{2}$ by the Gelfand-Naimark-Segal construction on Hilbert spaces $H_{1}$ and $H_{2}$ with unit cyclic vectors $\eta_{1}$ and $\eta_{2}$ respectively. Let $\left(\psi_{1}, K_{1}, \xi_{1}\right)$ and $\left(\psi_{2}, K_{2}, \xi_{2}\right)$ be the cyclic representation and the cyclic anti-representation derived from $g_{1}$ and $g_{2}$. The noncommutative Grothendieck inequality in this notation says that

$$
|\phi(x, y)| \leq\|\phi\|\left\|\left(\theta_{1} \oplus \theta_{2}\right)(x)\left(\eta_{1} \oplus \eta_{2}\right)\right\|\left\|\left(\psi_{1} \oplus \psi_{2}\right)(y)\left(\xi_{1} \oplus \xi_{2}\right)\right\|
$$

for all $x$ in $A$ and $y$ in $B$. The usual bilinear or sesquilinear form construction implies that there is a continuous linear operator $T$ from $K_{1} \oplus K_{2}$ into $H_{1} \oplus H_{2}$ with $\|T\| \leq\|\phi\|$ such that

$$
\phi(x, y)=\left\langle\left(\theta_{1} \oplus \theta_{2}\right)(x) T\left(\psi_{1} \oplus \psi_{2}\right)(y)\left(\xi_{1} \oplus \xi_{2}\right),\left(\eta_{1} \oplus \eta_{2}\right)\right\rangle
$$

for all $x$ in $A$ and $y$ in $B$. Writing $T$ as a 2 by 2 matrix of operators $\left(T_{i j}\right)$ arising from the direct sums $H_{1} \oplus H_{2}$ and $K_{1} \oplus K_{2}$ leads to four continuous bilinear forms $\phi_{1}, \ldots, \phi_{4}$ on $A \times B$ defined by

$$
\begin{aligned}
& \phi_{1}(x, y)=\left\langle\theta_{1}(x) T_{11} \psi_{1}(y) \xi_{1}, \eta_{1}\right\rangle, \\
& \phi_{2}(x, y)=\left\langle\theta_{1}(x) T_{12} \psi_{2}(y) \xi_{2}, \eta_{1}\right\rangle, \\
& \phi_{3}(x, y)=\left\langle\theta_{2}(x) T_{21} \psi_{1}(y) \xi_{1}, \eta_{2}\right\rangle \text { and } \\
& \phi_{4}(x, y)=\left\langle\theta_{2}(x) T_{22} \psi_{2}(y) \xi_{2}, \eta_{2}\right\rangle .
\end{aligned}
$$

The representation theorem for a continuous bilinear form on the Haagerup tensor product implies that

$$
\begin{aligned}
& \phi_{1} \text { is continuous on } A \otimes_{h} B \\
& \phi_{2} \text { is continuous on } A \otimes_{h} B^{o p}, \\
& \phi_{3} \text { is continuous on } A^{o p} \otimes_{h} B \text { and } \\
& \phi_{4} \text { is continuous on } A^{o p} \otimes_{h} B^{o p}
\end{aligned}
$$

all with $\left\|\phi_{j}\right\|_{h} \leq\|\phi\|$, where $A^{o p}$ is the reversed algebra. By the definition

$$
\phi=\phi_{1}+\phi_{2}+\phi_{3}+\phi_{4} \text {. }
$$

Proof of Theorem. That $\nu$ is an isometry was proved in Lemma 5.2 and that $\mu$ is norm reducing was proved in the initial discussion. To prove the inequality $\|u\| \leq 4\|\mu(u)\|$ for $u$ in $A^{* *} \otimes_{\gamma} B^{* *}$ let $u$ be in $A^{* *} \otimes B^{* *}$ with $\|u\|_{\gamma}=1$. By the Hahn-Banach Theorem there is a continuous bilinear form $\phi$ on $A^{* *} \times B^{* *}$ such that $\|\phi\|=1$ and $\phi(u)=1$. By the above discussion write

$$
\phi=\phi_{1}+\phi_{2}+\phi_{3}+\phi_{4}
$$

where $\phi_{j}$ are bilinear forms of norm no greater than one on $A^{* *} \otimes_{h} B^{* *}, A^{* *} \otimes_{h}$ $B^{* * o p}, A^{* * o p} \otimes_{h} B^{* *}, A^{* * o p} \otimes_{h} B^{* * o p}$ for $j=1, \ldots, 4$. Note that $A^{* * o p}=A^{o p * *}$. Let $\varepsilon>0$. If each of the von Neumann algebras $A^{* *}, A^{* * o p}, B^{* *}, B^{* * o p}$ is taken in the universal representation of $A, A^{o p}, B, B^{o p}$, then the bilinear forms $\phi_{j}$ may be 
weak ${ }^{*}$-approximated on the element $u$ by normal bilinear forms $\Phi_{j}$ with $\left\|\Phi_{j}\right\| \leq 1$ such that

$$
\left|\phi_{j}(u)-\Phi_{j}(u)\right|<\varepsilon \text {. }
$$

Because the bilinear forms $\Phi_{j}$ are normal they arise on their respective domains as lifts of bilinear forms on $A \times B$. Hence the definition of $\chi$ at the start of this section implies that for each $j, \Phi_{j}=\chi\left(\psi_{j}\right)$ where $\psi_{j}$ is the restriction of $\Phi_{j}$ to $A \times B$ in $A^{* *} \times B^{* *}$ is continuous with norm no greater than one on $A \otimes_{h} B, A \otimes_{h} B^{o p}, A^{o p} \otimes_{h}$ $B, A^{o p} \otimes_{h} B^{o p}$ for $j=1, \ldots, 4$, respectively. Let $\psi=\psi_{1}+\psi_{2}+\psi_{3}+\psi_{4}$. Then $\psi$ is a continuous bilinear form on $A \otimes_{\gamma} B$ with $\|\psi\| \leq 4$ because the projective norm dominates the four Haagerup type norms used above. Further

$$
\mu(u)(\psi)=\chi^{*} i(u)(\psi)=i(u)(\chi \psi)=(\chi \psi)(u)=\Phi(u)
$$

and

$$
|\mu(u)(\psi)-1|=|\Phi(u)-1|<4 \varepsilon .
$$

This implies that $\|\mu(u)\| \geq 1 / 4$ as required.

Finally we prove $\mu \nu=i_{A \otimes_{\gamma} B}$. Using the definitions of $\mu, \nu$ and $\chi$ gives the following equalities for all $\phi$ in $\left(A \otimes_{\gamma} B\right)^{*}=B i l(A \times B, \mathbb{C})$, all $a$ in $A$ and all $b$ in $B$ :

$$
\begin{gathered}
(\mu \nu)(a \otimes b)(\phi)=\left(\chi^{*} i \nu\right)(a \otimes b)(\phi)=(i \nu)(a \otimes b)(\chi \phi)=(\chi \phi)(\nu(a \otimes b)) \\
=(\chi \phi)\left(i_{A}(a) \otimes i_{B}(b)\right)=\phi(a \otimes b)=i_{A \otimes_{\gamma} B}(a \otimes b)(\phi) .
\end{gathered}
$$

This completes the proof of the theorem.

\section{Projective Banach Space and Haagerup norms}

In this section we prove the correct version of Itoh's result [14] on the equivalence of the projective Banach space norm and the Haagerup norm on the tensor products of $C^{*}$-algebras.

Theorem 6.1. If $A$ and $B$ are $C^{*}$-algebras, then the following conditions are equivalent:

(i) the Haagerup norm $\|\cdot\|_{h}$ is equivalent to the Banach space projective norm $\|\cdot\|_{\gamma}$,

(ii) either $A$ or $B$ is finite dimensional, or $A$ and $B$ are infinite dimensional and subhomogeneous.

The case where $A$ or $B$ is finite dimensional is trivial as both the tensors are then just Banach space isomorphic to a finite direct sum of the other algebra. The following lemmas will provide the proof of the non-trivial parts of the above theorem.

Lemma 6.2. Let $A$ and $B$ be infinite dimensional $C^{*}$-algebras. If for some $K>$ $0,\|u\|_{\gamma} \leq K\|u\|_{h}$ for all $u$ in the algebraic tensor product $A \otimes B$, then $A$ and $B$ are subhomogeneous with all their irreducible representations having dimension no greater than $16 K^{2}$.

Proof. Suppose that the identity map $j$ from $A \otimes_{h} B$ into $A \otimes_{\gamma} B$ is continuous and satisfies $\|j\| \leq K$. Then the second dual map $j^{* *}$ from $\left(A \otimes_{h} B\right)^{* *}$ into $\left(A \otimes_{\gamma} B\right)^{* *}$ also satisfies $\left\|j^{* *}\right\| \leq K$. This second dual map $j^{* *}$ restricted to $A^{* *} \otimes_{h} B^{* *}$ is essentially just the identity map $J\left(a^{* *} \otimes b^{* *}\right)=a^{* *} \otimes b^{* *}$ from $A^{* *} \otimes_{h} B^{* *}$ to 
$A^{* *} \otimes_{\gamma} B^{* *}$ as we now show. With the notation of Theorems 4.1 and 5.1 we have to show that $J=\mu^{-1} j^{* *} \zeta$ where $\zeta: A^{* *} \otimes_{h} B^{* *} \rightarrow\left(A \otimes_{h} B\right)^{* *}$ and $\mu: A^{* *} \otimes_{\gamma} B^{* *} \rightarrow$ $\left(A \otimes_{\gamma} B\right)^{* *}$ are the operators defined there by the separate ultraweak continuity of bilinear forms on $C^{*}$-algebras [12, Cor. 2.4]. The separate ultraweak continuity of $J, j^{* *}, \mu$ and $\zeta$ and the equality $\mu J=j^{* *} \zeta$ on $A \otimes B$ ensures that $\mu J=j^{* *} \zeta$ so that $J=\mu^{-1} j^{* *} \zeta$. Hence

$$
\|J\| \leq\left\|\mu^{-1}\right\|\left\|j^{* *}\right\|\|\zeta\| \leq 4 K
$$

If the von Neumann algebra $A^{* *}$ contains an isomorphic copy of $\mathbb{M}_{n}$ (not necessarily unitarily) for some positive integer $n$, then the other von Neumann algebra $B^{* *}$ contains a copy of $\ell_{n}^{\infty}$ since both are infinite dimensional. By the injectivity of the Haagerup norm $\mathbb{M}_{n} \otimes_{h} \ell_{n}^{\infty}$ embeds isometrically in $A^{* *} \otimes_{h} B^{* *}$ [20]. The embedding of $\mathbb{M}_{n} \otimes_{\gamma} \ell_{n}^{\infty}$ into $A^{* *} \otimes_{\gamma} B^{* *}$ is isometric, because there is a projection $P_{1} \otimes P_{2}$ of $A^{* *} \otimes_{\gamma} B^{* *}$ onto $\mathbb{M}_{n} \otimes_{\gamma} \ell_{n}^{\infty}$ with $\left\|P_{1} \otimes P_{2}\right\|=1$; here $P_{1}$ and $P_{2}$ are the norm 1 completely positive conditional expectations from $A^{* *}$ onto $\mathbb{M}_{n}$ and $B^{* *}$ onto $\ell_{n}^{\infty}$, respectively. With $e_{i j}$ the standard matrix units, by Lemma 3.1,

$$
\begin{aligned}
n^{\frac{1}{2}} & =\left\|\sum_{1}^{n} e_{1 j} \otimes e_{j j}\right\|_{h} \leq\left\|\sum_{1}^{n} e_{1 j} \otimes e_{j j}\right\|_{\gamma}=\left\|\sum_{1}^{n} e_{j 1} \otimes e_{j j}\right\|_{\gamma} \\
& \leq 4 K\left\|\sum_{1}^{n} e_{j 1} \otimes e_{j j}\right\|_{h} \\
& =4 K .
\end{aligned}
$$

The argument is symmetrical in $A$ and $B$, so neither $A^{* *}$ nor $B^{* *}$ can contain a type $\mathrm{I}_{n}$ factor with $n>16 K^{2}$. Thus $A^{* *}$ and $B^{* *}$ are finite type I von Neumann algebras with all their central direct summands of type $\mathrm{I}_{j}$ for $j \leq 16 K^{2}$. This implies that all the irreducible representations of $A$ and $B$ have dimension no greater than $16 K^{2}$, which proves the lemma.

Lemma 6.3. Let $A$ and $B$ be (infinite dimensional) subhomogeneous $C^{*}$-algebras. Then there is a constant $K$ such that $\|u\|_{\gamma} \leq K\|u\|_{h}$ for all $u \in A \otimes B$.

Proof. Since $A \otimes_{\gamma} B$ embeds isometrically in $A^{* *} \otimes_{\gamma} B^{* *}$ (Theorem 4.1) and $A \otimes_{h} B$ embeds in $A^{* *} \otimes_{h} B^{* *}[9],[5]$, the problem reduces to the case of homogeneous von Neumann algebrs because a subhomogeneous von Neumann algebra is a direct sum of homogeneous ones. Assume that $A=\mathbb{M}_{n}\left(A_{1}\right) \cong \mathbb{M}_{n} \otimes_{*} A_{1}$ and $B=$ $\mathbb{M}_{m}\left(B_{1}\right) \cong \mathbb{M}_{m} \otimes_{*} B_{1}$, where $A_{1}$ and $B_{1}$ are commutative $C^{*}$-algebras and $\otimes_{*}$ denotes the minimal $C^{*}$-tensor product. Now $A \otimes_{h} B$ is $\left(\mathbb{M}_{n} \otimes_{*} A_{1}\right) \otimes_{h}\left(\mathbb{M}_{m} \otimes_{*} B_{1}\right)$ so is Banach space equivalent to a direct sum of $n m$ copies of $A_{1} \otimes_{h} B_{1}$, and $A \otimes_{\gamma} B$ is $\left(\mathbb{M}_{n} \otimes_{*} A_{1}\right) \otimes_{\gamma}\left(M_{m} \otimes_{*} B_{1}\right)$ so is Banach space equivalent to a direct sum of $n m$ copies of $A_{1} \otimes_{\gamma} B_{1}$. Grothendieck's Theorem implies that $A_{1} \otimes_{h} B_{1}$ and $A_{1} \otimes_{\gamma} B_{1}$ are isomorphic up to Grothendieck's constant (see [21],[22]). Following these isomorphisms through shows that $\theta$ from $A \otimes_{h} B$ to $A \otimes_{\gamma} B$ is continuous. Taking $K=\|\theta\|$, we have the required result.

\section{OpERATOR SPACE PROJECTIVE NORM}

This section deals with the equivalence of the operator space projective norm with either the Haagerup norm or the Banach space projective norm. 
Lemma 7.1. Let $A$ and $B$ be $C^{*}$-algebras with $A$ commutative. Then $A \widehat{\otimes} B$ and $A \otimes_{\gamma} B$ are isomorphic with

$$
\|u\|_{\wedge} \leq\|u\|_{\gamma} \leq 2^{3 / 2}\|u\|_{\wedge}
$$

for all $u$ in $A \otimes B$.

Proof. The left hand inequality is well known and follows from $\|a \otimes b\|\left\|_{\wedge}=\right\| a\|\cdot\| b \|$ in $A \widehat{\otimes} B$ [4], [9] so we prove the right hand inequality. Let $u$ be in $A \otimes B$ with $\|u\|_{\wedge}=1$. By the Hahn-Banach Theorem and the representation of the dual of $A \otimes_{\gamma} B$ as the space of continuous bilinear forms on $A \times B$, choose a continuous bilinear form $F$ on $A \times B$ with $\|F\|=\|F\|_{\gamma}=1$ and $F(u)=\|u\|_{\gamma}$. The noncommutative Grothendieck inequality of Pisier and Haagerup [21], [22], [12] may be written in a representational form similar to the representation of a multilinear completely bounded operator ([16, p. 184, Remark 3.5(a) and (b)]) as follows (see also section 5). Corresponding to the continuous bilinear form $F$ there are cyclic representations $\phi_{1}$ and $\phi_{2}$ (and anti-representations $\psi_{1}$ and $\psi_{2}$ ) on Hilbert spaces $H_{1}$ and $H_{2}$ (and $K_{1}$ and $K_{2}$ ) with unit cyclic vectors $\xi_{1}$ and $\xi_{2}$ (and $\eta_{1}$ and $\eta_{2}$ ) and a continuous linear operator $T$ from $H_{2} \oplus K_{2}$ into $H_{1} \oplus K_{1}$ with $\|T\| \leq\|F\|_{\gamma}=1$ such that

$$
F(a, b)=\left\langle\left(\theta_{1} \oplus \psi_{1}\right)(a) T\left(\theta_{2} \oplus \psi_{2}\right)(b)\left(\xi_{2} \oplus \eta_{2}\right),\left(\xi_{1} \oplus \eta_{1}\right)\right\rangle
$$

for all $a$ in $A$ and $b$ in $B$.

Note that the notation has been changed slightly from ([16, p. 185]) to fit in with current completely bounded notation. The commutativity of $A$ ensures that $\pi=\theta_{1} \oplus \psi_{1}$ is a representation of $A$. Let

$$
F_{1}(a, b)=\left\langle\pi(a) T\left(\theta_{2}(b) \oplus 0\right)\left(\xi_{2} \oplus 0\right),\left(\xi_{1} \oplus \eta_{1}\right)\right\rangle
$$

and

$$
F_{2}(a, b)=\left\langle\pi(a) T\left(0 \oplus \psi_{2}(b)\right)\left(0 \oplus \eta_{2}\right),\left(\xi_{1} \oplus \eta_{1}\right)\right\rangle
$$

for $a$ in $A$ and $b$ in $B$. Since $\left\|\xi_{1} \oplus \eta_{1}\right\|=2^{\frac{1}{2}}$ and $\pi$ and $\theta_{2}$ are representations, it follows that $\left\|F_{1}\right\|_{h} \leq 2^{\frac{1}{2}}$ by the natural duality between $A \otimes_{h} B$ and the completely bounded bilinear forms $C B(A, B ; \mathbb{C})$ on $A \times B[7]$. Hence $\left\|F_{1}\right\|_{\wedge} \leq 2^{\frac{1}{2}}$, because $\|\cdot\|_{h} \leq\|\cdot\|_{\wedge}$ on $A \otimes B$ so the reverse inequality holds on the duals. Define the bilinear form $G$ on $A \times B\left(K_{2}\right)$ by

$$
G(a, x)=\left\langle\pi(a) T(0 \oplus x)\left(0 \oplus \eta_{2}\right),\left(\xi_{1} \oplus \eta_{1}\right)\right\rangle
$$

for all $a$ in $A$ and $x$ in $B\left(K_{2}\right)$. Again the natural duality between $A \otimes_{h} B\left(K_{2}\right)$ and the completely bounded bilinear forms on $A \times B\left(K_{2}\right)$ ensures that $\|G\|_{h} \leq 2^{\frac{1}{2}}$ as above. Hence $\|G\|_{\wedge} \leq 2^{\frac{1}{2}}$, where $G$ is considered as a continuous linear functional on $A \widehat{\otimes} B\left(K_{2}\right)$. Note that by the definition of $G$ and $F_{2}$,

$$
F_{2}=G .\left(I \otimes \psi_{2}\right)
$$

so that

$$
\left\|F_{2}\right\|_{\wedge} \leq\|G\|_{\wedge} \cdot\left\|I \otimes \psi_{2}\right\|_{\wedge} \leq 2^{\frac{1}{2}}
$$

by Lemma 3.2. Hence

$$
\|u\|_{\gamma}=F(u) \leq\left|F_{1}(u)\right|+\left|F_{2}(u)\right| \leq 2^{3 / 2}
$$

since $\|u\|_{\wedge}=1$. This completes the proof. 
These remarks are a reminder of results on embeddings involving the operator space projective tensor product. Recall that the standard identification between continuous bilinear forms $\phi$ on a tensor product $A \otimes B$ and continuous linear operators $\Phi$ from $A$ to $B^{*}$ given by $\phi(a \otimes b)=\Phi(a)(b)$ induces a (complete) isometry from $(A \widehat{\otimes} B)^{*}$ onto $C B\left(A, B^{*}\right)[4],[10]$. The duality of completely bounded operators implies that if $\Phi$ maps $A$ to $B^{*}$ is completely bounded, then $\Phi^{* *}$ maps $A^{* *}$ to $B^{* * *}$ with $\left\|\Phi^{* *}\right\|_{c b}=\|\Phi\|_{c b}$. Identifying $C B\left(A^{* *}, B^{* * *}\right)$ with $\left(A^{* *} \otimes_{h} B^{* *}\right)^{*}$ induces a (complete) isometry $\alpha$ from $(A \widehat{\otimes} B)^{*}$ into $\left(A^{* *} \widehat{\otimes} B^{* *}\right)^{*}$ given by $(\alpha \phi)\left(a^{* *} \otimes b^{* *}\right)=\Phi^{* *}\left(a^{* *}\right)\left(b^{* *}\right)$. Hence $\alpha^{*}$ is a (completely) contractive operator from $\left(A^{* *} \widehat{\otimes} B^{* *}\right)^{* *}$ into $(A \widehat{\otimes} B)^{* *}$. Let $\omega$ denote the norm reducing operator from $A^{* *} \widehat{\otimes} B^{* *}$ into $(A \widehat{\otimes} B)^{* *}$ given by composing $\alpha^{*}$ with the natural embedding of $A^{* *} \widehat{\otimes} B^{* *}$ into $\left(A^{* *} \widehat{\otimes} B^{* *}\right)^{* *}$.

Theorem 7.2. If $A$ and $B$ are $C^{*}$-algebras, the following conditions are equivalent:

(i) the operator space projective norm $\|\cdot\|_{\wedge}$ is equivalent to the Banach space projective norm $\|\cdot\|_{\gamma}$ on $A \otimes B$,

(ii) either $A$ or $B$ is subhomogeneous.

Proof. Suppose that $A$ is subhomogeneous. Since $A \otimes_{\gamma} B$ embeds isometrically in $A^{* *} \otimes_{\gamma} B^{* *}$ (Theorem 5.1) and $A \widehat{\otimes} B$ embeds isometrically in $A^{* *} \widehat{\otimes} B^{* *}$ [8], the problem reduces to the case when $A$ is a homogeneous von Neumann algebra because a subhomogeneous von Neumann algebra is a direct sum of homogeneous ones. Assume that $A=\mathbb{M}_{n}(C)$, where $C$ is a commutative von Neumann algebra. Then $A \widehat{\otimes} B$ is Banach space isomorphic to a direct sum of $n^{2}$ copies of $C \widehat{\otimes} B$ since $\left(e_{i j} \otimes 1\right)(C \widehat{\otimes} B)$ is isometrically embedded in $A \widehat{\otimes} B$ for all matrix units $e_{i j}$ in $\mathbb{M}_{n}$ as there is a conditional expectation from $A$ onto $C$. By Lemma 7.1, $C \widehat{\otimes} B$ is equivalent to $C \otimes_{\gamma} B$ so that the $\|\cdot\|_{\gamma}$ and $\|\cdot\|_{\wedge}$ norms are equivalent on $A \otimes B$.

Conversely suppose that there is a $K>0$ such that $\|u\|_{\gamma} \leq K\|u\|_{\wedge}$ for all $u$ in $A \otimes B$. The map $\omega$ from $A^{* *} \widehat{\otimes} B^{* *}$ into $(A \widehat{\otimes} B)^{* *}$ is norm reducing (see Lemma 7.1) so that $\|\omega(u)\|_{* *} \leq\|u\|_{\wedge}$ and the map $\mu$ from $A^{* *} \otimes_{\gamma} B^{* *}$ into $\left(A \otimes_{\gamma} B\right)^{* *}$ satisfies $\|u\|_{\gamma} \leq 4\|u\|_{* *}$ for all $u$ in $A^{* *} \otimes B^{* *}$ (Theorem 4.1). Here $\|\cdot\|_{\wedge}$ and $\|\cdot\|_{\gamma}$ denote the norms in $A^{* *} \widehat{\otimes} B^{* *}$ and $A^{* *} \widehat{\otimes}_{\gamma} B^{* *}$, respectively, and $\|\cdot\|_{* *}$ is the relevant second dual norm. In this notation the supposition at the second dual level becomes

$$
\|\mu(u)\|_{* *} \leq K\|\omega(u)\|_{* *}
$$

so that

$$
\|u\|_{\gamma} \leq 4\|\mu(u)\|_{* *} \leq 4 K\|\omega(u)\|_{* *} \leq 4 K\|u\|_{\wedge}
$$

for all $u$ in $A^{* *} \otimes B^{* *}$.

If for some positive integer $n, A^{* *}$ and $B^{* *}$ contain a copy of $\mathbb{M}_{n}$, then $\mathbb{M}_{n} \widehat{\otimes} \mathbb{M}_{n}$ embeds isometrically into $A^{* *} \widehat{\otimes} B^{* *}$ and $\mathbb{M}_{n} \otimes_{\gamma} \mathbb{M}_{n}$ embeds isometrically into $A^{* *} \otimes_{\gamma} B^{* *}$. Using Lemma 3.3 it follows that

$$
n=\left\|\sum e_{j 1} \otimes e_{j 1}\right\|_{\gamma} \leq 4 K\left\|\sum e_{j 1} \otimes e_{j 1}\right\|_{\wedge}=4 K n^{\frac{1}{2}} .
$$

Hence $n \leq 16 K^{2}$. Thus either $A^{* *}$ or $B^{* *}$ does not contain a type $\mathrm{I}_{n}$ factor for $n>16 K^{2}$ so that $A$ or $B$ is subhomogeneous. This proves the theorem.

The remainder of this section deals with the equivalence of the Haagerup norm and the operator space projective norm on $A \otimes B$. 
Lemma 7.3. Let $A$ and $B$ be $C^{*}$-algebras. If $\|\cdot\|_{h}$ is equivalent to $\|\cdot\|_{\wedge}$ on $A \otimes B$, then $A$ or $B$ are subhomogeneous.

Proof. Suppose that $A$ and $B$ are non-subhomogeneous $C^{*}$-algebras and let $n$ be a positive integer. By a lemma of R.R. Smith [24, Lemma 2.7] and [13] there are completely positive contractions $\rho_{A}: \mathbb{M}_{n} \rightarrow A, \sigma_{A}: A \rightarrow \mathbb{M}_{n}, \rho_{B}: \mathbb{M}_{n} \rightarrow B, \sigma_{B}:$ $B \rightarrow \mathbb{M}_{n}$ such that

$$
\left\|\sigma_{A} \rho_{A}-i d\right\|<1 / 2 n \text { and }\left\|\sigma_{B} \rho_{B}-i d\right\|<1 / 2 n \text { on } \mathbb{M}_{n} \text {. }
$$

Let $a_{i}=\rho_{A}\left(e_{i 1}\right)$ and $b_{i}=\rho_{B}\left(e_{1 i}\right)$ for $1 \leq i \leq n$. Let $\left\{H_{A}, \pi_{A}, V_{A}\right\}$ and $\left\{H_{B}, \pi_{B}, V_{B}\right\}$ be the Stinespring representations of $\sigma_{A}$ and $\sigma_{B}$, respectively, where $\pi_{A}$ and $\pi_{B}$ are representations of $A$ and $B$ on $H_{A}$ and $H_{B}$, and $V_{A}$ and $V_{B}$ are contractions from $\mathbb{C}^{n}$ into $H_{A}$ and $H_{B}$ such that

$$
\sigma_{A}(a)=V_{A}^{*} \pi_{A}(a) V_{A} \quad(a \in A)
$$

and

$$
\sigma_{B}(b)=V_{B}^{*} \pi_{B}(b) V_{B}(b \in B)
$$

[19, pp. 43-45].

Let $x=\sum_{1}^{n} a_{i} \otimes b_{i}$ be in $A \otimes B$. Since $\pi_{A} \otimes \pi_{B}$ is norm reducing by the projectivity of $\|\cdot\|_{\wedge}$ and since left and right multiplication is continuous on $\widehat{\otimes}$,

$$
\begin{aligned}
\|x\| & \geq\left\|V_{A}^{*} \otimes V_{B}^{*}\right\| \cdot\left\|\sum \pi_{A}\left(a_{i}\right) \otimes \pi_{B}\left(b_{i}\right)\right\|_{\wedge} \cdot\left\|V_{A} \otimes V_{B}\right\| \\
& \geq\left\|\sum \sigma_{A}\left(a_{i}\right) \otimes \sigma_{B}\left(b_{i}\right)\right\|_{\wedge} .
\end{aligned}
$$

The inequalities

$$
\left\|\sigma_{A}\left(a_{i}\right)-e_{i 1}\right\|=\left\|\sigma_{A} \rho_{A}\left(e_{i 1}\right)-e_{i 1}\right\| \leq\left\|\sigma_{A} \rho_{A}-i d\right\|<1 / 2 n
$$

and

$$
\left\|\sigma_{B}\left(b_{i}\right)-e_{1 i}\right\|<1 / 2 n
$$

imply that

$$
\begin{aligned}
& \left|\left\|\sum \sigma_{A}\left(a_{i}\right) \otimes \sigma_{B}\left(b_{i}\right)\right\|_{\wedge}-\left\|\sum e_{i 1} \otimes e_{1 i}\right\|_{\wedge}\right| \\
\leq & \left\|\sum \sigma_{A}\left(a_{i}\right) \otimes \sigma_{B}\left(b_{i}\right)-\sum e_{i 1} \otimes e_{1 i}\right\|_{\wedge} \\
\leq & \left\|\sum \sigma_{A}\left(a_{i}\right) \otimes\left(\sigma_{B}\left(b_{i}\right)-e_{1 i}\right)\right\|_{\wedge}+\left\|\sum\left(\sigma_{A}\left(a_{i}\right)-e_{i 1}\right) \otimes e_{1 i}\right\|_{\wedge} \\
\leq & \sum\left\|\sigma_{A}\left(a_{i}\right)\right\| \cdot\left\|\sigma_{B}\left(b_{i}\right)-e_{1 i}\right\|+\sum\left\|\sigma_{A}\left(a_{i}\right)-e_{i 1}\right\| \cdot\left\|e_{1 i}\right\| \\
\leq & 2 n / 2 n=1 .
\end{aligned}
$$

Thus

$$
\|x\| \geq\left\|\sum \sigma_{A}\left(a_{i}\right) \otimes \sigma_{B}\left(b_{i}\right)\right\|_{\wedge} \geq\left\|\sum e_{i 1} \otimes e_{1 i}\right\|_{\wedge}-1=n-1
$$

by Lemma 3.3 .

The complete positivity of $\rho_{A}$ and $\left\|\rho_{A}\right\|=1$ implies that

$$
\left\|\sum a_{i} a_{i}^{*}\right\|=\left\|\sum \rho_{A}\left(e_{i 1}\right) \rho_{A}\left(e_{i 1}\right)^{*}\right\| \leq\left\|\sum \rho_{A}\left(e_{i 1} e_{i 1}^{*}\right)\right\| \leq\left\|\sum e_{i 1} e_{1 i}\right\|=1
$$

by the general Cauchy-Schwarz inequality for completely positive maps.

Similarly

$$
\left\|\sum b_{i}^{*} b_{i}\right\| \leq 1
$$


The definition of the Haagerup norm on $A \otimes B$ implies that

$$
\|x\|_{h} \leq\left\|\sum a_{i} a_{i}^{*}\right\|^{\frac{1}{2}} \cdot\left\|\sum b_{i}^{*} b_{i}\right\|^{\frac{1}{2}}=1 .
$$

This proves that $\|\cdot\|_{h}$ and $\|\cdot\|_{\wedge}$ are not equivalent on $A \otimes B$ and so proves the Lemma.

Theorem 7.4. Let $A$ and $B$ be $C^{*}$-algebras. Then the following are equivalent:

(i) the Haagerup norm $\|\cdot\|_{h}$ is equivalent to the operator space projective norm $\|\cdot\|_{\wedge}$,

(ii) either $A$ or $B$ is finite dimensional, or $A$ and $B$ are infinite dimensional and subhomogeneous.

Proof. Since $\|\cdot\|_{h} \leq\|\cdot\|_{\wedge} \leq\|\cdot\|_{\gamma}$, (ii) $\Rightarrow$ (i) is trivial using Theorem 6.1. If (i) holds then $A$ or $B$ is subhomogeneous by Lemma 7.3 which in turn implies that $\|\cdot\|_{\wedge}$ is equivalent to $\|\cdot\|_{\gamma}$ (Theorem 7.2). Thus $\|\cdot\|_{h}$ is equivalent to $\|\cdot\|_{\gamma}$. Applying Theorem 6.1 again we have the condition (ii).

\section{REFERENCES}

1. R.J. Archbold and C.J.K. Batty, $C^{*}$-tensor norms and slice maps, J. London Math. Soc. (2) 22 (1980) 127-138. MR 81j:46090

2. D.P. Blecher, Geometry of the tensor product of $C^{*}$-algebras, Math. Proc. Camb. Phil. Soc. 104 (1988), 119-127. MR 89g:46094

3. D.P. Blecher, Tensor products of operator spaces II, Can. J. Math. 44 (1992), 75-90. MR 93e: 46084

4. D.P. Blecher and V.I. Paulsen, Tensor product of operator spaces, J. Funct. Anal. 99 (1991), 262-292. MR 93d:46095

5. D.P. Blecher and R.R. Smith, The dual of the Haagerup tensor product, J. London Math. Soc. 45 (1992), 126-144. MR 93h:46078

6. E. Christensen and A.M. Sinclair, A survey of completely bounded operators, Bull. London Math. Soc. 21 (1989), 417-448. MR 91b:46051

7. E.G. Effros and A. Kishimoto, Module maps and Hochschild-Johnson cohomology, Indiana Univ. Math. J. 36 (1987), 257-276. MR 89b:46068

8. E.G. Effros and Z-J. Ruan, On approximation properties for operator spaces, International J. Math. 1 (1990), 163-187. MR 92g:46089

9. E.G. Effros and Z-J. Ruan, Self duality for the Haagerup tensor product and Hilbert space factorization, J. Funct. Anal. 100 (1991), 257-284. MR 93f:46090

10. E.G. Effros and Z-J. Ruan, A new approach to operator spaces, Bull. Can. Math. Soc. 34 (1991), 329-337. MR 93a:47045

11. E.G. Effros and Z-J. Ruan, Operator convolution algebras. An approach to Quantum groups. (preprint).

12. U. Haagerup, The Grothendieck inequality for bilinear forms on $C^{*}$-algebras, Advances in Math. 56 (1985), 93-116. MR 86j:46061

13. T. Huruya and J. Tomiyama, Completely bounded maps of $C^{*}$-algebras, J. Operator Theory 10 (1983), 141-152. MR 85f:46108a

14. T. Itoh, The Haagerup type cross norm on $C^{*}$-algebras, Proc. Amer. Math. Soc. 109 (1990), 689-695. MR 90m:46096

15. B.E. Johnson, R.V. Kadison and J.R. Ringrose, Cohomology of operator algebras III, Reduction to normal cohomology, Bull. Soc. Math. France 100 (1972),73-96. MR 47:7454

16. S. Kaijser and A.M. Sinclair, Projective tensor products of $C^{*}$-algebras, Math. Scand. 55 (1984), 161-187. MR 86m:46053

17. A. Kumar, Involution and the Haagerup tensor product (preprint).

18. M. Mathieu, Generalising elementary operators, Semesterbericht Funktionalanalysis SS88, Tübingen (1988), 133-153.

19. V.I. Paulsen, Completely bounded maps and dilations, Pitman Research Notes in Math. 146 Longman, 1986. MR 88h:46111 
20. V.I. Paulsen and R.R. Smith, Multilinear maps and tensor norms on operator systems, J. Funct. Anal. 73 (1987), 258-276. MR 89m:46099

21. G. Pisier, Grothendieck's theorem for non-commutative $C^{*}$-algebras with an appendix on Grothendieck's constant, J. Funct. Anal. 29 (1978), 397-415. MR 80j:47027

22. G. Pisier, Factorization of linear operators and the geometry of Banach spaces, CBMS Series No. 60, A.M.S. Providence, R.I., 1986. MR 88a:47020

23. A.M. Sinclair and R.R. Smith, Hochschild Cohomology of von Neumann Algebras, Lecture Notes Series 203, London Mathematical Society, 1995. MR 96d:46094

24. R.R. Smith, Completely bounded maps between $C^{*}$-algebras, J. London Math. Soc. 27 (1983), 157-166. MR 84g:46086

25. R.R. Smith, Completely bounded module maps and the Haagerup tensor product, J. Funct. Anal. 102 (1991), 156-175. MR 93a:46115

26. M. Takesaki, Theory of operator algebras I, Springer-Verlag, Berlin, 1979. MR 81e:46038

Department of Mathematics, Rajdhani College (University of Delhi), Raja Garden, New DelHI-110015, INDIA

Department of Mathematics and Statistics, University of Edinburgh, James Clerk Maxwell Building, King's Buildings, Mayfield Road, Edinburgh EH9 3JZ, Scotland

E-mail address: allan@maths.ed.ac.uk 